\title{
DESARROLLO DE TOBERAS DE COHETE EN MATERIAL ABLATIVO DE MATRIZ POLIMÉRICA REFORZADA ESTRUCTURALMENTE CON FIBRAS DE VIDRIO Y CARBONO*
}

\author{
Jorge Maldonado Villa \\ Julián Portocarrero Hermann² \\ Esteban Quitian Barrios ${ }^{3}$ \\ Jorge Andrés Alarcón Orduz ${ }^{3}$ \\ William David Boada ${ }^{3}$
}

\section{Resumo}

Se desarrollaron materiales compuestos de matriz polimérica para toberas en cohetes; con dos zonas: una térmica para soportar elevadas temperaturas de combustión y una estructural que resista los esfuerzos generados. La zona térmica se elaboró con materiales de comportamiento ablativo, probados a temperaturas de hasta $2950^{\circ} \mathrm{C}$ durante dos minutos, los cuales fueron reforzados con polvo cerámico y/o subproducto de la industria siderúrgica (cerámico 2). Con base en los resultados obtenidos del comportamiento de térmico y de conductividad térmica del material ablativo, se construyó la zona estructural en materiales compuestos fibroreforzados (vidrio y carbono) con matriz polimérica. Los moldes se diseñaron en SolidWorks y se fabricaron por prototipado en ABS rápido y se elaboraron machos disgregables en poliuretano. En la zona térmica se usó un procedimiento de vaciado con vibración con diferentes tipos de matrices (poliéster, vinilester, caucho silicona) y de laminación para la zona estructural. Se evaluó el desempeño mediante simulación de las condiciones reales de operación mediante llama de oxicorte y quemador de ACPM, observando el efecto en la pérdida de peso, espesor, diferencial de temperatura, cambio de propiedades mecánicas, etc.; encontrándose un positivo comportamiento del sistema con matriz de resina vinilester reforzado con polvo cerámico 1 y buenos resultados con cerámico 2, así como una estabilidad en la zona estructural construida con fibras de vidrio y carbono. Substituir todo o texto de orientação deste Template pelo conteúdo de seu trabalho. Inserir o texto com no máximo 1.500 caracteres (200 palavras), sem uso de parágrafos. Deve conter, de maneira concisa, o objetivo, a metodologia, os resultados e as conclusões do trabalho.

Palavras-chave: Tobera para cohete; Materiales compuestos particulados; Materiales compuestos fibro-reforzados; Material compuesto ablativo.

\section{ROCKET NOZZLE DEVELOPMENT IN ABLATIVE MATERIAL POLYMER MATRIX STRUCTURALLY REINFORCED WITH FIBRE GLASS AND CARBON}

\begin{abstract}
Polymer matrix composites for rocket nozzles developed; two areas: Thermal for high combustion temperatures and external structure to withstand the stresses generated. The thermal zone ablative materials behavior, tested at temperatures up to $2950^{\circ} \mathrm{C}$ for two minutes, reinforced ceramic powder and / or dust-product of the steel industry (ceramic 2). Based on the results of the behavior of thermal conductivity and thermal ablative material the structural zone built in fiber-reinforced composite materials (glass and carbon) with polymeric matrix. The molds were designed in SolidWorks and fabricated by rapid prototyping $A B S$, disintegrating males were manufactured in polyurethane. In the thermal zone emptying procedure vibration with different matrices (polyester, vinylester, silicone rubber) and rolling for the structural zone was used. Performance by simulating real operating conditions was assessed using oxyfuel flame and burner ACPM, loss of weight, thickness, temperature differential change of mechanical properties, determined etc .; found a positive performance of the system with matrix resin with ceramic powder 1 vinylester good results with ceramic 2 and in the structural area built with fiberglass and carbon.

Keywords: Rocket nozzle; Particulate materials composites; Fibro-reinforced materials composites; Composite ablative material.

1 Doctor en Ingeniería con Énfasis en Ingeniería de Materiales. Ingeniero de Materiales, Tecnólogo Químico, Docente - Investigador, Sector Aeroespacial y Defensa, Escuela Militar de Aviación "Marco Fidel Suarez" - Fuerza Aérea Colombiana, Cali/Valle del Cauca/Colombia.

2 Ingeniero con Estudios Doctorales en Ingeniería con Énfasis en Ingeniería de Materiales. Ingeniero Mecánico, Docente - Investigador, Sector Aeroespacial y Defensa, Escuela Militar de Aviación "Marco Fidel Suarez", Fuerza Aérea Colombiana, Cali/Valle del Cauca/Colombia.

3 Estudiante de Ingeniería Mecánica, Estudiante de Último año - proyectante, Sector Aeroespacial y Defensa, Escuela Militar de Aviación "Marco Fidel Suarez", Fuerza Aérea Colombiana, Cali/Valle del Cauca/Colombia.
\end{abstract}




\section{INTRODUCCIÓN}

Un cohete es un vehículo o aeronave que obtiene su empuje por la reacción de la expulsión rápida de gases de combustión desde un motor cohete; los componentes básicos de cualquier motor de cohete son: el inyector, la cámara de combustión y la tobera de escape de gases o boquilla. La cámara de combustión es el lugar donde se transforma la energía potencial (energía interna del combustible) en energía cinética de los gases producto de la combustión, esta debe ser ligera y relativamente pequeña, en el caso de la tobera usada en este proyecto que es convergente divergente (tipo Laval) esta es la parte inicial de la tobera y la salida de los gases o boquilla es la parte final ver Figura 1. En este proyecto se da a conocer el desarrollo de un material ablativo para toberas de cohetes de órbita baja a partir de materiales compuestos de matriz polimérica y reforzamiento con partículas cerámicas, en el cual se evalúa el efecto ablativo de tres tipos de matrices de resina poliéster, viniléster y caucho silicona y dos tipos de reforzantes particulados polvo cerámico (feldespato-caolín) y la escoria de aluminio, adicionalmente a las mezclas se les agrego un $3 \%$ de fibra de vidrio corta a $3 \mathrm{~mm}$ de longitud, para disminuir la tendencia a fisuración que sufren este tipo de materiales por los esfuerzos térmicos durante su operación. Este proyecto que hace parte del programa inicial de desarrollo de un cohete de órbita baja en la Fuerza Aérea Colombiana con que se pretende fortalecer la escuela del conocimiento en diferentes áreas del campo aeroespacial. De esta tecnología desarrollada, se destaca que estas son aproximadamente un $90 \%$ más livianas que aquellas hechas con aleaciones metálicas.

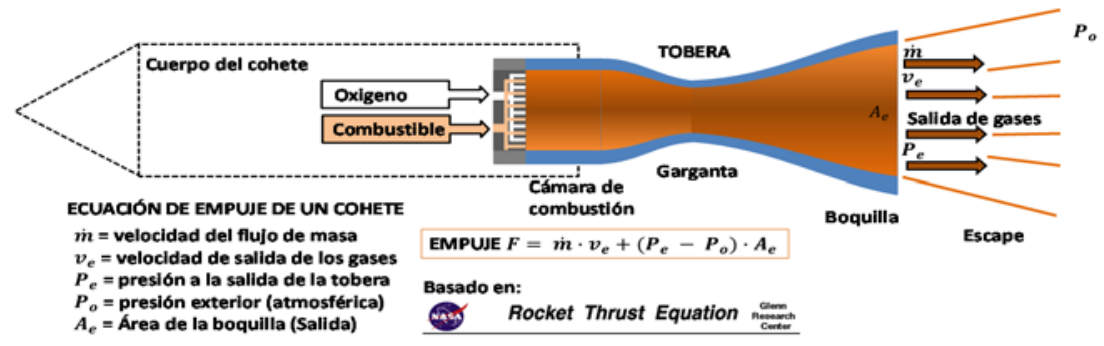

Figura 1. Cohete y ecuación de empuje de un cohete $^{1}$

En esta investigación se analizó el comportamiento ablativo de toberas a escala menor (1:5) fabricadas en material compuesto ablativo, por medio de pruebas de llama a alta temperatura en tiempos de exposición de 120 segundos, con los cuales de determino la pérdida de peso del sistema, la perdida de espesor del material en la zona térmica, el perfil de aislamiento y la modificación estereomicroestructural del material ablativo. En los resultados se aprecia que un desecho industrial como la escoria de aluminio puede aportar propiedades equiparables a las obtenidas con reforzantes particulados de polvo cerámico de uso convencional en la industria de materiales compuestos, dejando abierta la posibilidad en este proyecto de analizar la capacidad de aporte estructural y térmico de materiales ablativos reforzados con escorias siderúrgicas o de acerías que se utilizó en estudios previos con muy buenos resultados. Para el desarrollo de este proyecto se recurrió a diferentes fuentes de información desde los proyectos anteriores desarrollados en la Escuela Militar de Aviación (seis años trabajo en el tema), hasta patentes registradas en la OMPI, pasando por trabajos publicados por NASA y España.

\footnotetext{
${ }^{1}$ Basado en 1. Benson [1], Tom. Rocket Thrust Equation. GRC.NASA [Internet]. USA. [actualizado Jun 12 2014]. Citado 4 mar 2015]. Disponible en: http://www.grc.nasa.gov/WWW/k-12/airplane/rockth.html
} 


\section{MATERIAL ABLATIVO}

Los materiales ablativos son materiales que se usan en la zona interna de las toberas Jae-Seok [3], Alhama [4], Morozov [5], Schmidt [6], como sistema de protección para la zona estructural de la tobera, debido a las altas temperaturas y a la erosión térmica que se generan en este sistema; el mecanismo de funcionamiento de un material ablativo segun Maldonado et al [7] está descrito por las siguientes 5 funciones (ver Figura 2). Actualmente los materiales ablativos se elaboran a base de materiales compuestos particulados endurecidos por dispersión, en la mayoría de los casos son de matriz polimérica, con algunos fibrorreforzantes.

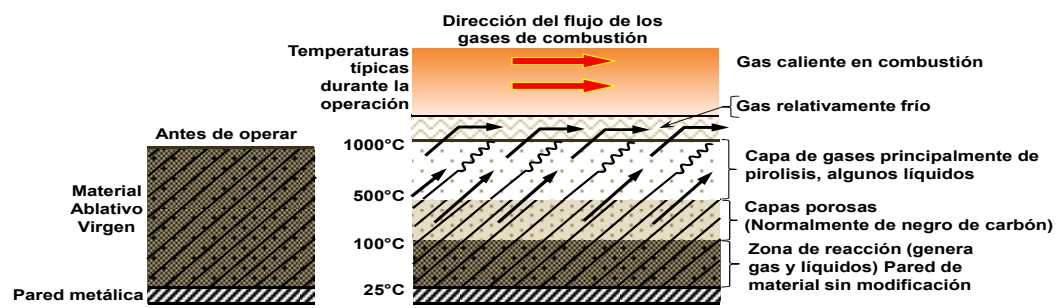

Figura 2. Zonas de un material ablativo durante la operación de un cohete ${ }^{2}$.

a) Autoconsumible: esta es una de las principales funciones que se le exigen a un material ablativo, en la que se busca que el material cambie de estado, la capa superficial genera un gas durante el tiempo de vuelo o funcionamiento de la tobera, el cual absorbe y retiene una cantidad de calor, y además ayuda a incrementar la resistencia al desgaste Furness [10].

b) Aislante Térmico: la mezcla de material ablativo además al convertirse en gas consumiendo calor latente por el cambio de estado y calor sensible aumento de temperatura, el gas generado actúa como aislante térmico, forma un colchón o coraza aislante, contra la onda de choque del agente del calentamiento dado su baja conductividad térmica y el arrastre de calor (evacuación de los gases y materia fundida), adicionalmente el material compuesto restante de la tobera ayuda como aislante térmico, el material quemado parcialmente (capa en contacto con la combustión de los gases) adquiere una consistencia esponjosa (aislante por la porosidad e inquemados cerámicos) por la combustión del polímero y sinterización del material particulado, además el material no quemado está elaborado con materiales que poseen una abaja conductividad térmica.

c) Refractario: Los sistemas ablativos deben de cumplir con la condición de soportar altas temperaturas las cuales pueden ser superiores a los $2200^{\circ} \mathrm{C}$, lo cual se presenta en los materiales ablativos por las dos condiciones anteriores.

d) Resistencia a la Erosión Térmica: estos materiales a pesar de ser Autoconsumibles también deben de ser resistentes a la erosión térmica Bo Chen [11], debido a las altas velocidades que se requieren para generar una fuerza de empuje en el sistema, esto se logra por la generación de gases que forman un colchón protector y por la sinterización de las partículas cerámicas, con lo que se genera un desgaste por erosión térmica paulatino y controlado.

e) Resistente a Esfuerzo Térmico: La mezcla ablativa también debe soportar los altos esfuerzos térmicos de dilatación que se producen en el sistema de tal manera que la formación de fisuras y microfisuras en el mismo se generen de

\footnotetext{
${ }^{2}$ Basado en: Sutton [2], George P. Rocket Propulsion Elements. Séptima edición. New York, USA. John Wiley \& Sons, Inc. 2001, p 561-563.
} 
manera lo menos posible y de forma tardía en la última etapa de uso de la tobera. Lo anterior se logra al introducir microfibras de alta resistencia.

\section{MATERIALES Y MÉTODOS}

\subsection{Metodología}

El diseño metodológico sigue un patrón descriptivo-correlacional; así pues, que describe el comportamiento de los materiales seleccionados y sometidos a las pruebas, teniendo como referencia parámetros y protocolos ya establecidos en proyectos, pruebas previas y en la bibliografía consultada. El trabajo se concentra en un programa experimental para la evaluación del material ablativo en la zona térmica de la tobera, mediante exposición a llama, se evaluaron tres tipos de matrices y dos reforzantes particulados; fijando en un $3 \%$ el nivel de fibras cortas de vidrio $E$ de $3 \mathrm{~mm}$ de longitud orientadas al azar como reforzante (disminuir la fisuración por esfuerzos térmicos) y la zona estructural (basado en los trabajos de Bastidas et al [14], Lizcano et al [25], Quiñones et al [16], Maldonado et al [9,17-22], Portocarrero et al [21,22]; evaluando las variables respuesta pérdida de peso, perdida de espesor, perfil de aislamiento y modificación estereomicroestructural, ver Tabla 1.

Tabla 1. Programa experimental

\begin{tabular}{|c|c|c|c|c|c|}
\hline \multirow{2}{*}{ MUESTRA } & \multicolumn{3}{|c|}{ ZONA TÉRMICA } & \multirow{2}{*}{$\begin{array}{c}\text { ZONA } \\
\text { ESTRUCTURAL }\end{array}$} & \multirow{2}{*}{$\begin{array}{l}\text { VARIABLE DE } \\
\text { RESPUESTA }\end{array}$} \\
\hline & MATRIZ & REFORZ & VTE & & \\
\hline 1 & Resina viniléster & Escoria metálica & \multirow{6}{*}{$\begin{array}{l}3 \% \text { fibras } \\
\text { cortas de } \\
\text { vidrio } \mathrm{E} \text { de } \\
3 \mathrm{~mm} \text { de } \\
\text { longitud } \\
\text { orientadas } \\
\text { al azar }\end{array}$} & \multirow{6}{*}{$\begin{array}{l}\text { Laminación } \\
\text { manual }\end{array}$} & \multirow{6}{*}{$\begin{array}{l}\text { Comportamiento Térmico } \\
\text { y Ablativo (pérdida de } \\
\text { peso, la diferencia de } \\
\text { espesores, perfiles } \\
\text { térmicos, cambios y } \\
\text { estereomorfología } \\
\text { superficial) }\end{array}$} \\
\hline 2 & Resina viniléster & Polvo cerámico & & & \\
\hline 3 & Resina poliéster & Escoria metálica & & & \\
\hline 4 & Resina poliéster & Polvo cerámico & & & \\
\hline 5 & Caucho silicona & Escoria metálica & & & \\
\hline 6 & Caucho silicona & Polvo cerámico & & & \\
\hline
\end{tabular}

Todas las toberas curadas a $50^{\circ} \mathrm{C}$ durante 8 horas.

\subsection{Elaboración de Toberas}

En la zona estructural basado en los resultados obtenidos por Maldonado et al [8], se definió como un sistema multicapa de fibras de carbono/ fibra de vidrio tipo voland/ fibra de carbono/ fibra de vidrio tipo voland/ fibra de carbono, con orientaciones de $0^{\circ}-90^{\circ}$ embebidas en matrices de resina vinil éster, sistema estructural de acuerdo a las evaluaciones en este estudio permite soportar presiones en la cámara de combustión entre 10 y 15 atmosferas, Las toberas una ves construidas fueron sometidas a un proceso

El proceso de fabricación consistió en elaborar toberas en una escala menor de 1:5, de acuerdo a diseños previos de Bello y Bedoya (2008) y Nakka [23] en el que el molde se elaboró a partir de una impresión en una máquina de prototipado rápido, marca Dimensión serie U-print plus que consiste en un sistema de molde y contra molde, este último por las dificultades de extracción, presentadas durante el moldeo de la tobera fueron elaborados a partir de poliuretano expandido rígido, el cual fue fácilmente disgregable, después del curado de la resina. El proceso consistió en elaborar las mezclas por medio de un método de vaciado de la mezcla de la zona térmica (ver Figura 3) según la Tabla 1, con tiempos de vaciado inferiores a 10 minutos. Una vez polimerizada la mezcla se efectuó un proceso de laminación manual alrededor de la misma para la colocación de la zona estructural, las cuales finalmente fueron sometidas a un proceso de termo curado de $50^{\circ} \mathrm{C}$ durante 8 horas. 


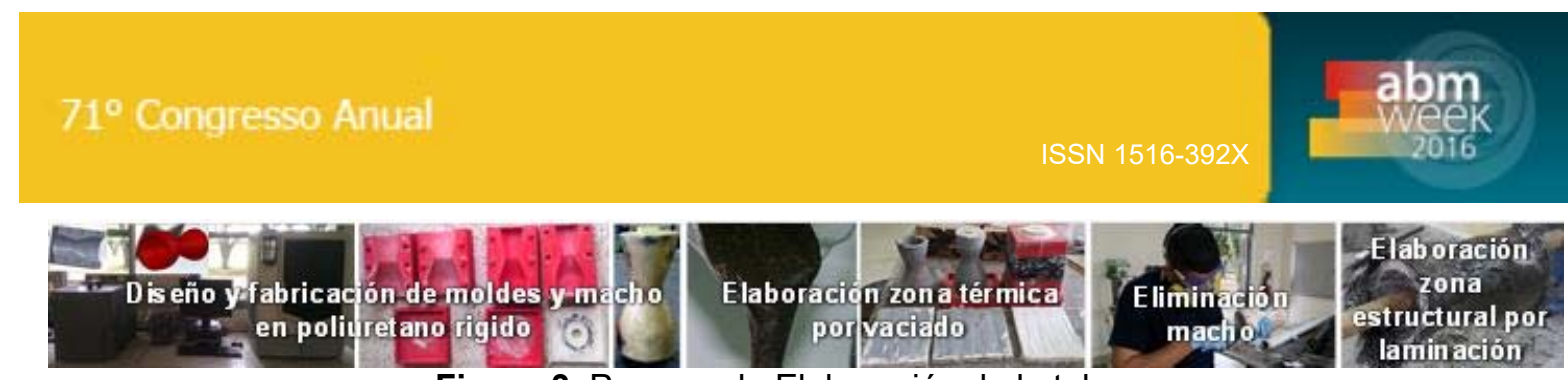

Figura 3. Proceso de Elaboración de la toberas.

\subsection{Prueba de Llama}

En las pruebas de llama las toberas elaboradas en escala menor de 1:5, se sometieron a pruebas de llama con un quemador de ACPM marca Beckett Sunteo A2VA-7116, potencializado con un compresor, para obtener temperaturas de llama superiores a $1500^{\circ} \mathrm{C}$, ver Figura 3 y 4 , durante 120 segundos para evaluar la pérdida de peso, el perfil de aislamiento térmico, se midio las temperaturas durante el proceso con una cámara termográfica (FLIR E40); la perdida de espesor y la variación superficial estructural y estereomicroestructural del sistema ablativo, para lo cual se utilizó un esteromicroscopio de $7 X$ a 90X. Es de resaltar que el subproducto industrial de la fundición de aluminio por su alto contenido de amoniaco generado por los tratamientos de extracción de aluminio metálico, fue sometido a un proceso de lavado y acondicionamiento para reducir los efectos en el sistema e incrementar la resistencia en cada una de las matrices.
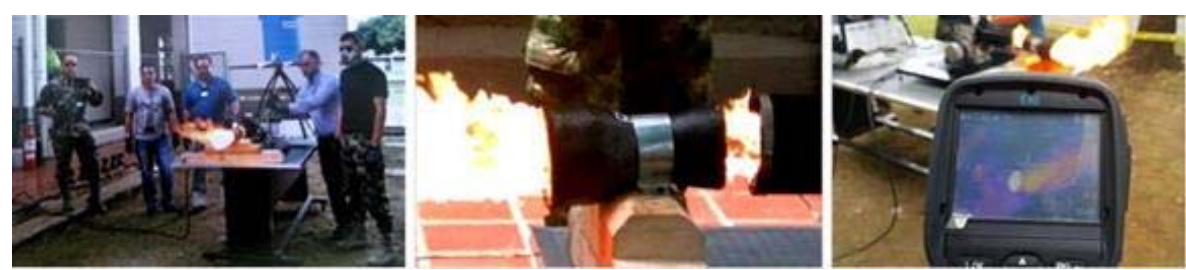

Figura 3. Prueba de llama.

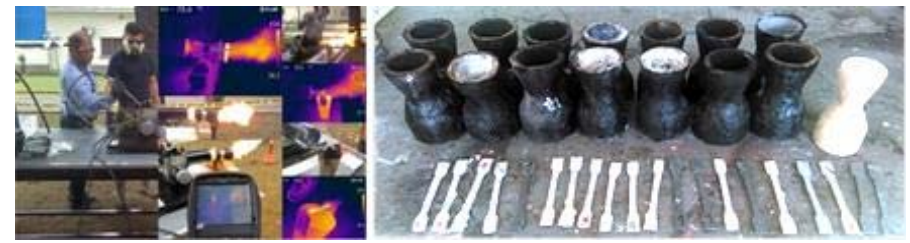

Figura 4. Izquierda termografía. Derecha toberas después de la prueba de llama; um macho em poliuretano y probetas para ensayo de tensión.

\section{RESULTADOS Y DISCUSIÓN}

\subsection{Resultados y Análisis por Pérdida de Peso}

Como se observa en la Tabla 2 la pérdida de peso menor se presentó en la combinación de resina viniléster con escoria y la mayor pérdida de peso fue la de caucho silicona con escoria (38,08 mayor), al observarlo por tipo de matriz la resina viniléster experimento menor tipo de pérdida de peso, la resina poliéster tuvo un incremento del $5,39 \%$ de pérdida de peso y el caucho silicona un $20,85 \%$ con respecto a la matriz de resina viniléster, en cuanto al tipo de reforzante presentó un ligero mejor resultado el polvo cerámico (ver Tabla 3). Comparando estos resultados con los obtenidos por Acuña et al [24] y los de Lizcano et al [15,25], en los que utilizaron escoria siderúrgica y polvo cerámico con matrices similares con un soplete de oxiacetilénico, que los resultados en cuanto a matrices fueron similares, en cuanto a los refuerzos ellos usaron escoria siderúrgica la cual presenta mejor comportamiento a altas temperaturas, por eso a ellos les dio mejor resultado con la escoria. 
Tabla 2. Pérdida de peso de peso después de la prueba

\begin{tabular}{|c|c|c|c|c|c|c|}
\hline SERIE & COMPOSICIÓN & $\begin{array}{c}\text { PESO INICIAL } \\
\text { (g) }\end{array}$ & $\begin{array}{c}\text { PESO } \\
\text { FINAL (g) }\end{array}$ & $\begin{array}{l}\text { PERDIDA DE } \\
\text { PESO (g) }\end{array}$ & $\begin{array}{c}\text { PROMEDIO } \\
\text { PERDIDA PESO (g) }\end{array}$ & $\begin{array}{l}\text { \% PERDIDA PESO } \\
\text { RESP. A LA MEJOR }\end{array}$ \\
\hline IA & \multirow{2}{*}{$\begin{array}{c}\text { Resina Vinilester + } \\
\text { escoria }\end{array}$} & 767,62 & 739,07 & 28,55 & \multirow{2}{*}{29,005} & \multirow{2}{*}{$0,00 \%$} \\
\hline $\mathrm{IB}$ & & 772,54 & 743,08 & 29,46 & & \\
\hline IIA & \multirow{2}{*}{$\begin{array}{l}\text { Resina poliester }+ \\
\text { polvo cerámico }\end{array}$} & 712,97 & 684,32 & 28,65 & \multirow{2}{*}{31,725} & \multirow{2}{*}{$-9,38 \%$} \\
\hline IIB & & 726,04 & 691,24 & 34,8 & & \\
\hline IIIA & \multirow{2}{*}{$\begin{array}{l}\text { Resina vinilester + } \\
\text { polvo cerámico }\end{array}$} & 728,49 & 700,65 & 27,84 & \multirow{2}{*}{32,095} & \multirow{2}{*}{$-10,65 \%$} \\
\hline IIIB & & 723,08 & 686,73 & 36,35 & & \\
\hline IVA & \multirow{3}{*}{$\begin{array}{l}\text { Resina poliester + } \\
\text { escoria }\end{array}$} & 588,24 & 559,63 & 28,61 & \multirow{3}{*}{32,853} & \multirow{3}{*}{$-13,27 \%$} \\
\hline IVB & & 696,68 & 662,06 & 34,62 & & \\
\hline IVC & & 658,48 & 623,15 & 35,33 & & \\
\hline VA & \multirow{2}{*}{$\begin{array}{c}\text { Cauchosilicona + } \\
\text { escoria }\end{array}$} & 619,5 & 580,69 & 38,81 & \multirow{2}{*}{40,05} & \multirow{2}{*}{$-38,08 \%$} \\
\hline VB & & 528,14 & 486,85 & 41,29 & & \\
\hline$X A$ & \multirow{2}{*}{$\begin{array}{c}\text { Cauchosilicona + polvo } \\
\text { cerámico }\end{array}$} & 498,11 & 462,61 & 35,5 & \multirow{2}{*}{37,145} & \multirow{2}{*}{$-28,06 \%$} \\
\hline XB & & 530,5 & 491,71 & 38,79 & & \\
\hline
\end{tabular}

Tabla 3. Pérdida de peso por tipo de matriz y por tipo de refuerzo

\begin{tabular}{|l|c|c|l|c|c|}
\hline \multicolumn{1}{|c|}{ MATRIZ } & $\begin{array}{c}\text { PROM. PERDIDA PESO } \\
\mathbf{( g )}\end{array}$ & $\begin{array}{c}\text { \% PERD. PESO } \\
\text { RESPECTO AL } \\
\text { MEJOR }\end{array}$ & REFUERZO & $\begin{array}{c}\text { PROMEDIO } \\
\text { PERDIDA } \\
\text { PESO (g) }\end{array}$ & $\begin{array}{c}\text { \% PERDIDA } \\
\text { PESO RESPECTO } \\
\text { A LA MEJOR }\end{array}$ \\
\hline Viniléster & 30,55 & $0,00 \%$ & Polvo cerámico & 33,66 & $0,00 \%$ \\
\hline Poliéster & 32,29 & $-5,39 \%$ & Escoria de Al & 33,97 & $-0,93 \%$ \\
\hline Caucho silicona & 38,60 & $-20,85 \%$ & \multicolumn{2}{|l}{} \\
\cline { 1 - 5 } & &
\end{tabular}

\subsection{Resultados y Análisis por Gradiente Térmico}

En la Tabla 4 se aprecia que la tobera del mayor gradiente térmico medido durante la prueba, la cual es una medida indirecta de la capacidad de aislamiento, fue la combinación de resina poliéster con escoria, ahora se puede decir que en general todos presentaron muy buen gradiente térmico lo que reafirma el uso de material compuesto fibroreforzado para la zona estructural dado que esta no se afectaría. Los resultados con los obtenidos por Acuña et al [24], los de Lizcano et al [15,25], las de Chen et al [11] y los de Alhama et al [4], confirman estos resultados. En la Tabla 5 aparecen los comparativos con las matrices y los refuerzos, se encuentra un ligero mejor comportamiento en la resina poliéster como matriz y en el polvo cerámico; aunque estos valores no presentan una diferencia significativa. Las temperaturas internas medidas en las toberas (Tabla 6) después de la prueba de llama indican que el colchón de gases generados, el cambio de estado de los algunos componentes (adsorben calor latente) y el desprendimiento de algunas partículas, están contribuyendo al proceso ablativo esperado.
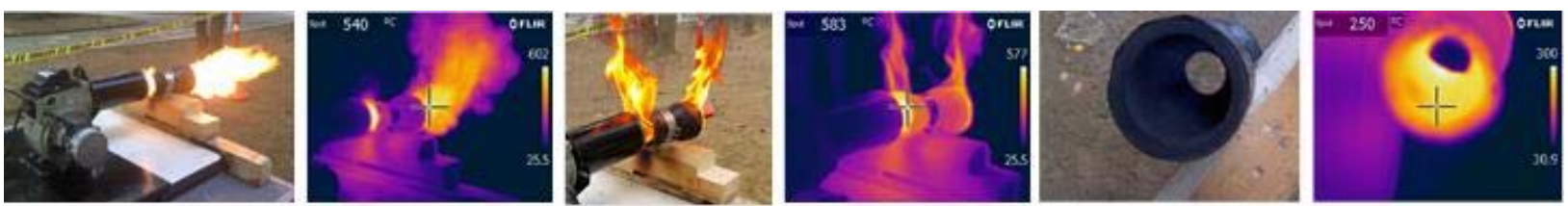

Figura 5. Toma de temperaturas de la tobera durante la prueba y al terminar. 
Tabla 4. Gradiente térmico medido durante la de la prueba

\begin{tabular}{|c|c|c|c|c|c|c|}
\hline \multirow[t]{2}{*}{ SERIE } & \multirow[t]{2}{*}{ COMPOSICIÓN } & \multicolumn{2}{|c|}{$\begin{array}{c}\text { TEMPERATURA DURANTE LA } \\
\text { QUEMA }\left({ }^{\circ} \mathrm{C}\right)\end{array}$} & \multirow{2}{*}{$\begin{array}{l}\text { GRADIENTE } \\
\text { TÉRMICO }\end{array}$} & \multirow{2}{*}{$\begin{array}{l}\text { PROM. } \\
\text { GRADIENTE }\end{array}$} & \multirow{2}{*}{$\begin{array}{c}\text { \% PROM. } \\
\text { GRADIENTE TÉR. }\end{array}$} \\
\hline & & INLET & SUPERFICIE & & & \\
\hline IA & \multirow{2}{*}{$\begin{array}{c}\text { Resina Vinilester + } \\
\text { escoria }\end{array}$} & 1600 & 127 & 1473 & \multirow{2}{*}{1480,5} & \multirow{2}{*}{$-0,57 \%$} \\
\hline IB & & 1600 & 112 & 1488 & & \\
\hline IIA & \multirow{2}{*}{$\begin{array}{c}\text { Resina poliester }+ \\
\text { polvo cerámico }\end{array}$} & 1600 & 119 & 1481 & \multirow{2}{*}{1480,5} & \multirow{2}{*}{$-0,57 \%$} \\
\hline IIB & & 1600 & 120 & 1480 & & \\
\hline IIIA & \multirow{2}{*}{$\begin{array}{c}\text { Resina vinilester }+ \\
\text { polvo cerámico }\end{array}$} & 1600 & 104 & 1496 & \multirow{2}{*}{1484,0} & \multirow{2}{*}{$-0,34 \%$} \\
\hline IIIB & & 1600 & 128 & 1472 & & \\
\hline IVA & \multirow{3}{*}{$\begin{array}{l}\text { Resina poliester }+ \\
\text { escoria }\end{array}$} & 1600 & 116 & 1484 & \multirow{3}{*}{1489,0} & \multirow{3}{*}{$0,00 \%$} \\
\hline IVB & & 1600 & 105 & 1495 & & \\
\hline IVC & & 1600 & 112 & 1488 & & \\
\hline VA & \multirow{2}{*}{$\begin{array}{c}\text { Cauchosilicona + } \\
\text { escoria }\end{array}$} & 1600 & 130 & 1470 & \multirow{2}{*}{1478,0} & \multirow{2}{*}{$-0,74 \%$} \\
\hline VB & & 1600 & 114 & 1486 & & \\
\hline $\mathrm{XA}$ & \multirow{2}{*}{$\begin{array}{l}\text { Cauchosilicona + } \\
\text { polvo cerámico }\end{array}$} & 1600 & 138 & 1462 & \multirow{2}{*}{1473,5} & \multirow{2}{*}{$-1,04 \%$} \\
\hline $\mathrm{XB}$ & & 1600 & 115 & 1485 & & \\
\hline
\end{tabular}

Tabla 5. Gradiente térmico medido durante la de la prueba para el tipo de matriz y tipo de reforzante

\begin{tabular}{|l|c|c|c|c|c|}
\hline \multicolumn{1}{|c|}{ MATRIZ } & $\begin{array}{c}\text { GRAD. } \\
\text { TÉRMICO }\left({ }^{\circ} \mathrm{C}\right)\end{array}$ & $\begin{array}{c}\text { \% GRAD. TÉR. } \\
\text { RESPECTO AL MEJOR }\end{array}$ & REFUERZO & $\begin{array}{c}\text { GRAD. } \\
\text { TÉRMICO }\left({ }^{\circ} \mathrm{C}\right)\end{array}$ & $\begin{array}{c}\% \text { GRADIENTE TÉR. } \\
\text { RESPETO AL MEJOR }\end{array}$ \\
\hline Viniléster & 1482,25 & $-0,17 \%$ & Polvo cerámico & 1479,33 & $-0,21 \%$ \\
\hline Poliéster & 1484,75 & $0,00 \%$ & Escoria de Al & 1482,50 & $0.00 \%$ \\
\hline Caucho silicona & 1475,75 & $-0,61 \%$ & \multicolumn{3}{|c}{} \\
\cline { 1 - 3 } & & &
\end{tabular}

Tabla 6.Temperatura interna de la tobera después de la prueba de llama

\begin{tabular}{|c|c|c|c|c|c|}
\hline \multirow{3}{*}{ SERIE } & \multicolumn{2}{|c|}{ DESPUES DE LA QUEMA } & \multirow{3}{*}{ SERIE } & \multicolumn{2}{|c|}{ DESPUES DE LA QUEMA } \\
\hline & \multirow{2}{*}{ COMPOSICIÓN } & TEMPERATURA ${ }^{\circ} \mathrm{C}$ & & \multirow{2}{*}{ COMPOSICIÓN } & TEMPERATURA ${ }^{\circ} \mathrm{C}$ \\
\hline & & INLET & & & INLET \\
\hline IA & \multirow{2}{*}{$\begin{array}{c}\text { Resina Vinilester + } \\
\text { escoria }\end{array}$} & 433 & IVA & \multirow{3}{*}{$\begin{array}{c}\text { Resina poliester + } \\
\text { escoria }\end{array}$} & 382 \\
\hline IB & & 303 & IVB & & 363 \\
\hline$\| A$ & \multirow{2}{*}{$\begin{array}{c}\text { Resina poliester + } \\
\text { polvo cerámico }\end{array}$} & 364 & IVC & & 415 \\
\hline$\| \mathrm{B}$ & & 384 & VA & \multirow{2}{*}{$\begin{array}{c}\text { Cauchosilicona + } \\
\text { escoria }\end{array}$} & 429 \\
\hline IIIA & \multirow{2}{*}{$\begin{array}{c}\text { Resina vinilester + } \\
\text { polvo cerámico }\end{array}$} & 373 & VB & & 413 \\
\hline IIIB & & 383 & $X A$ & \multirow{2}{*}{$\begin{array}{l}\text { Cauchosilicona + } \\
\text { polvo cerámico }\end{array}$} & 380 \\
\hline & & & XB & & 332 \\
\hline
\end{tabular}

\subsection{Resultados y Análisis Pérdida de Espesor}

Para determinar el espesor se tomaron diferentes medidas interiores y exteriores en puntos específicos antes y después de la pruebas de llama, en la Tabla 7 se muestran los resultados, donde la tobera del mejor resultado fue la combinación de resina viniléster con escoria, aunque se puede decir que en general todas los sistemas tuvieron un buen comportamiento, las matrices de caucho silicona presentaron la mayor pérdida de espesor, adicionalmente el material quedo escamado (ver Figura 6), por lo cual las medidas tomadas no son tan precisas, dado que la perdida de material real es más grande. Al observar la Tabla 8 en la cual se compara los resultados por tipo de matriz y por tipo de reforzante, se aprecia un contraste con el resultado anterior, un mejor resultado fue para la matriz de poliéster y como reforzante el polvo cerámico. Es de anotar que la diferencia entre las matrices de poliéster y viniléster, así como para los reforzantes sobre estas matrices fue mínima. 
Tabla 7. Resultados de la pérdida de espesor después de la prueba de llama

\begin{tabular}{|c|c|c|c|c|c|c|c|c|}
\hline \multirow{3}{*}{ SERIE } & \multirow{3}{*}{ COMPOSICIÓN } & \multicolumn{2}{|c|}{ ESPESOR INICIAL (mm) } & \multicolumn{2}{|c|}{ ESPESOR INICIAL (mm) } & \multirow{3}{*}{$\begin{array}{l}\text { PÉRDIDA } \\
\text { ESPESOR } \\
(\mathrm{mm})\end{array}$} & \multirow{3}{*}{$\begin{array}{l}\text { PÉRDIDA } \\
\text { PROM. } \\
\text { ESP. (mm) }\end{array}$} & \multirow{3}{*}{$\begin{array}{l}\text { \% PROM. } \\
\text { PÉRD. ESPE. } \\
\text { RESP. MEJOR }\end{array}$} \\
\hline & & INLET & OUTLET & INLET & OUTLET & & & \\
\hline & & PROM. & PROM. & PROM. & PROM. & & & \\
\hline IA & \multirow{2}{*}{$\begin{array}{c}\text { Resina Vinilester } \\
+ \text { escoria }\end{array}$} & 12,238 & 12,386 & 12,052 & 11,84 & 0,366 & \multirow{2}{*}{0,2620} & \multirow{2}{*}{0,0000} \\
\hline IB & & 10,53 & 11,262 & 10,466 & 11,01 & 0,158 & & \\
\hline$I I A$ & \multirow{2}{*}{$\begin{array}{l}\text { Resina poliester } \\
+ \text { polvo cerámico }\end{array}$} & 11,02 & 10,798 & 10,546 & 10,122 & 0,575 & \multirow{2}{*}{0,4830} & \multirow{2}{*}{$-0,8435$} \\
\hline IIB & & 8,728 & 10,548 & 8,502 & 9,992 & 0,391 & & \\
\hline IIIA & \multirow{2}{*}{$\begin{array}{l}\text { Resina vinilester } \\
+ \text { polvo cerámico }\end{array}$} & 10,704 & 11,566 & 9,622 & 11,26 & 0,694 & \multirow{2}{*}{0,7550} & \multirow{2}{*}{$-1,8817$} \\
\hline IIIB & & 9,97 & 12,346 & 9,102 & 11,582 & 0,816 & & \\
\hline IVA & \multirow{3}{*}{$\begin{array}{l}\text { Resina poliester } \\
+ \text { escoria }\end{array}$} & 8,792 & 10,606 & 7,758 & 10,11 & 0,765 & \multirow{3}{*}{0,5007} & \multirow{3}{*}{$-1,8817$} \\
\hline IVB & & 10,824 & 11,408 & 9,938 & 11,114 & 0,59 & & \\
\hline IVC & & 11,268 & 11,596 & 11,064 & 11,506 & 0,147 & & \\
\hline VA & \multirow{2}{*}{$\begin{array}{l}\text { Caucho silicona } \\
\text { + escoria }\end{array}$} & 11,892 & 12,114 & 11,22 & 9,462 & 1,662 & \multirow{2}{*}{2,2490} & \multirow{2}{*}{$-7,5840$} \\
\hline VB & & 9,926 & 11,5 & 8,25 & 7,504 & 2,836 & & \\
\hline$X A$ & \multirow{2}{*}{$\begin{array}{l}\text { Caucho silicona + } \\
\text { polvo cerámico }\end{array}$} & 9,268 & 10,934 & 8,078 & 10,878 & 0,623 & \multirow{2}{*}{1,1825} & \multirow{2}{*}{$-3,5134$} \\
\hline XB & & 11,952 & 12,014 & 10,632 & 9,85 & 1,742 & & \\
\hline
\end{tabular}

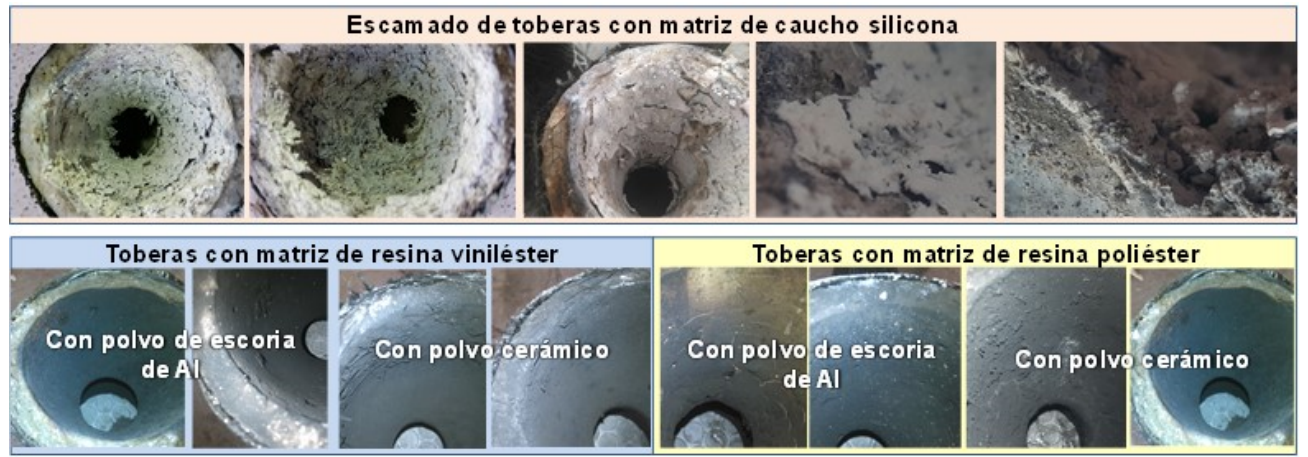

Figura 6. Estado de las superficies interiores de la tobera después de la prueba de llama

Tabla 8. Pérdida de espesor después de la prueba con base en el tipo de matriz y del tipo de refuerzo.

\begin{tabular}{|l|r|r|l|r|r|}
\hline \multicolumn{1}{|c|}{ MATRIZ } & $\begin{array}{c}\text { PERD. PROM. ESP. } \\
(\mathbf{m m})\end{array}$ & $\begin{array}{c}\text { \% PERDIDA } \\
\text { ESPESOR }\end{array}$ & REFUERZO & $\begin{array}{c}\text { PERD. PROM. } \\
\text { ESP. (mm) }\end{array}$ & $\begin{array}{c}\text { \% PERDIDA } \\
\text { ESPESOR }\end{array}$ \\
\hline Viniléster & 0,51 & $-0,034$ & Cerámico & 0,81 & 0,000 \\
\hline Poliéster & 0,49 & 0,000 & Escoria de Al & 1,00 & $-0,244$ \\
\hline Caucho Silicona & 1,72 & $-2,488$ & & &
\end{tabular}

\subsection{Análisis Óptico y Estéreomicroscopico de la Superficie Expuesta}

De acuerdo a los análisis realizados en las diferentes toberas por observación directa y estéreomicroscopia realizadas después de la exposición a la llama, se aprecia especialmente que los materiales de matriz de resina poliéster y resina viniléster, presentaron un comportamiento esperado de tipo ablativo, en cuanto a la formación de zona superficial porosa con aparente micro sinterización de partículas cerámicas (ver Figuras 6, 7 y 8). Lo cual indica la formación de una capa gaseosa de los componentes de la matriz, esta toma parte de calor de los gases en combustión para cambiar de estado (solido a gaseoso por pirolisis) absorbiendo una buena cantidad de energía calorífica al cambiar de estado, calor latente, como se mencionó en el análisis del gradiente térmico (Figura 7), incrementando el flujo de gases, además esta capa gaseosa ayuda a incrementar la resistencia al desgaste erosivo. 


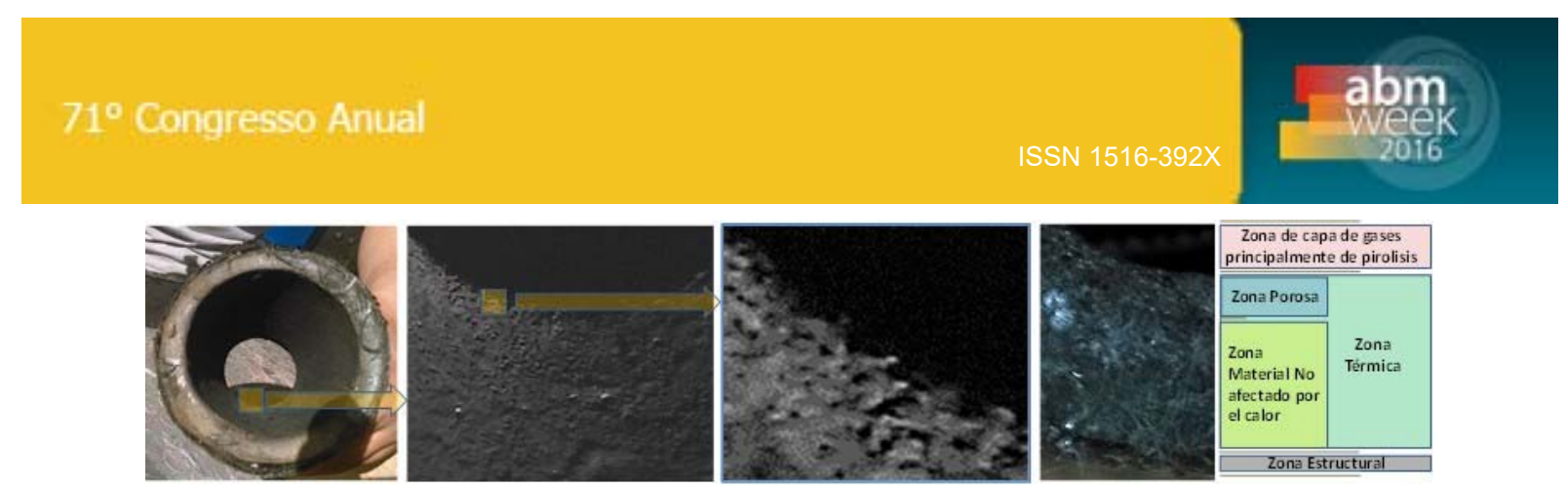

Figura 7. Formación de microporos en la superficie dela zona térmica de expuesta a la llama.

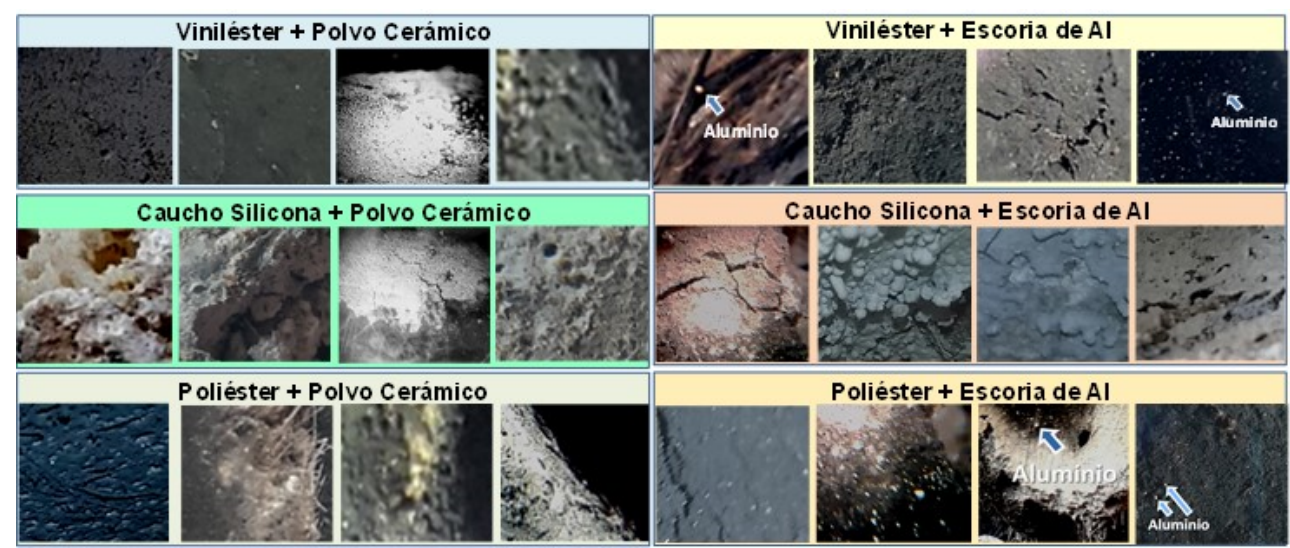

Figura 8. Estéreomicroscopias de las superficies dela zona térmica de expuesta a la llama.

En el caso de los materiales con polvo de escoria de aluminio se encontró una generación de segregaciones de aluminio metálico y un mayor nivel de microfisuraciones las cuales probablemente fueron causadas por estas microsegregaciones y por la presencia de sales volátiles como amoniaco (producto de los procesos de recuperación de aluminio que se le hace a la escoria en los talleres de fundición), estas probablemente fueron la cusa de un pequeño porcentaje mayor de porosidad alrededor de un $5 \%$ en la superficie de estas toberas. En el caso de las toberas que se utilizaron matriz de caucho silicona se presentó un alto nivel de descamación, fisuración y poros de mayor tamaño, lo cual no es muy deseable en el desarrollo de materiales ablativos, dado la posible formación de elementos erosivos, que afecten el desempeño del resto del material ablativo; adicionalmente se encontró un alto nivel de combustión de esta matriz, después de realizada la prueba de llama.

\section{CONCLUSIONES}

Se logró establecer el comportamiento que los materiales compuestos utilizados presentaron un comportamiento de tipo ablativo:

> Cambio de estado y formación de gases por pirolisis de la zona superficial expuesta, consumiendo y arrastrando una buena cantidad de calor, según los resultados de estéreomicroscopia y gradiente térmico.

$>$ Se presentó un efecto de aislamiento térmico generado por el colchón de gases y el consumo de calor latente durante la pirolisis de la matriz, esto se puede asumir por la temperatura alcanzada en el interior inmediatamente después de finalizada la prueba de llama, no superaron los $435^{\circ} \mathrm{C}$ y la temperatura de llama fue mayor de $1600^{\circ} \mathrm{C}$.

$>$ Se presentó una formación de un material microporoso en la superficie, esperado, con micro sinterizaciones de las partículas cerámicas, el cual cumple un efecto aislante; además dado que los componentes de los materiales compuestos utilizados son malos conductores del calor, los 
materiales compuestos usados presentaron un buen comportamiento de aislamiento térmico; esto se evidenció durante el estudio del gradientermico.

$>$ Las toberas fueron expuestas a temperaturas superiores a los $1600^{\circ} \mathrm{C}$ durante 120 segundos, no presentando perdidas de peso o espesor significativos. No se presentó además una erosión térmica apreciable en las matrices de resina viniléster, ni en las de resina poliéster; el resultado fue menos significativo en las de matriz de cauco silicona donde se presentó una escamación del material en la superficie.

$>$ El nivel de fisuración encontrado fue bajo, se apreciaron microfisuras superficiales en especial en las matrices reforzadas con escoria de aluminio, pero ninguna atraviesa la zona térmica ni compromete estructuralmente la tobera.

La matriz elastoplástica de caucho silicona presentó menores resultados que las matrices de resina poliéster y de resina viniléster, aunque los resultados no fueron los mejores, mostró un comportamiento ablativo.

Las diferencias en el comportamiento de las matrices de resina poliéster y viniléster en cuanto a sus propiedades ablativas, no fueron significativas, dado que la resina poliéster es más económica se recomienda su utilización en este tipo de materiales.

La escoria de Al funcionó bien como reforzante del material ablativo, aunque según estudios anteriores comparativos es menor el desempeño con respecto al uso de escoria siderúrgica.

Los resultados obtenidos con los materiales compuestos ablativos desarrollados en EMAVI fueron muy Buenos, al compararlos con las aplicaciones y los rangos de temperaturas de los materiales para toberas de cohete que aparecen en Huzel et al. [26], Hisaichi Ohnabe et al. [27], Jae-Seok et al. [3], Schmidt [6], Bo Chen [11] y otros autores.

\section{REFERENCIAS}

1 Benson, Tom. Rocket Thrust Equation. GRC.NASA [Internet]. USA. [actualizado Jun 12 2014]. Citado 4 mar 2015]. Disponible en: http://www.grc.nasa.gov/WWW/k12/airplane/rockth.html

2 Sutton, George P. Rocket Propulsion Elements. Séptima edición. New York, USA. John Wiley \& Sons, Inc. 2001, p 561-563.

3 Jae-Seok Yoo, Sang-Eui Lee, Chun-Gon Kim. Prediction of mechanical behavior of spatially reinforced composites for kick motor nozzle. [Internet]. Elsevier. Korea. Composite Structures 54 (2001) 57-65. [Agosto 11 de 2001, última consulta: 6 marzo de 2015]. Disponible en: www.elsevier.com/locate/compstruct

4 Alhama, Francisco; Campo, Antonio. Network simulation of the rapid temperature changes in the composite nozzle wall of an experimental rocket engine during a ground firing test. España -USA Applied Thermal Engineering 23 (2003) 37-47.

5 Morozov, E.V., Pitot De La Beaujardiere, J.F.P. Numerical simulation of the dynamic thermostructural response of a composite rocket nozzle throat, Australia -Sur Africa. Composite Structures 91 (2009) 412-420

6 Schmidt, Donald L. Ablative Materials. [Internet]. Illinois Institute of Technology. Website CONTRAILS.IIT.EDU; USA; Marzo 3 de 2009. [Marzo 3 de 2009, última consulta: 13 marzo de 2015]. Disponible en:

http://contrails.iit.edu/DigitalCollection/1961/ASDTR61-322article49.pdf

7 Maldonado Villa, Jorge; Portocarrero Hermann, Julian. Desarrollo de una Tobera a Escala Menor en Material Compuesto Ablativo para de Cohete de Órbita Baja. En: 
Colombia. Memorias Evento: Segunda Versión. Semana del Saber Científico Militar. Escuela Militar de Cadetes “José María Córdova”. Bogotá. Colombia. Septiembre 2012. Portocarrero Hermann, Julián; Maldonado Villa, Jorge. Evaluación del comportamiento térmico de materiales compuestos de matriz polimérica en prototipos de toberas para cohetes de órbita baja. Colombia. Revista Científica "General José María Córdova" ISSN: 1900-6586 ed.: Editorial Planeta v.12 fasc.13 (2014) p.275 - 290,

9 Portocarrero Hermann, Julián; Maldonado Villa, Jorge; Evaluación del comportamiento mecánico de los materiales compuestos en toberas para cohetes de órbita baja Colombia. 2014. Revista Colombiana de Materiales. ISSN: 2256-1013 p.303 - 309 v.5 Furness, Justin. Materials Information Service: Thermosetting Composites Processing. USA. AZOM Materials. 2001. [Diciembre 2001, última consulta: 13 marzo de 2015] Internet: www.azom.com/Details.asp?ArticleID=352.

11 Bo Chen, Li-Tong Zhang, Lai-Fei Cheng, Xin-Gang Luan; Erosion resistance of needled carbon/carbon composites exposed to solid rocket motor plumes. [Internet]. China. ELSEVIER. CARBON 47 (2009) 1474-1479. [Marzo 20 de 2009, última consulta: 13 marzo de 2015]. Internet: www.elsevier.com/locate/carbon

12 Congdon, William. Advanced Ablative Insulators and Methods of Making Them. Reinforced, filled silicones and carbon phenolics are laser-milled to final shapes [Internet]. 1a ed. [Johnson Space Center] Houston, Texas, USA. September 2005 [Septiembre de 2005, última consulta: 11 marzo de 2015]. Disponible en internet: <http://www.techbriefs.com/component/content/257?task=view>.

13 Dow-Corning-Corporation, Dow Corning ${ }^{\circledR} 93104$ Ablative Material USA. [Internet]. 1a ed. [Ellsworth Adhesives] Washington, Marzo 1999 [Marzo de 1999, última consulta: 14 marzo de 2015]. Internet: <http://207.250.200.229:8080/1/doc?id=3728>.

14 Bastidas Rendon, Jairo Y Gerena Romero, Jefferson. Estudio y caracterización de los materiales para la construcción de una tobera para los cohetes de la FAC. Santiago de Cali, Colombia, 2009. Proyecto de Grado Ingeniero Mecánico, Escuela Militar De Aviación. Facultad de Ingeniería Mecánica. [Noviembre 2009].

15 Lizcano G. Y.; Vásquez B. C.; Maldonado Villa J.; Rodríguez Adaime, C.; Portocarrero Hermann, J.; Paredes Muñoz, R. Comportamiento Térmico y Estructural de Materiales para Tobera de Cohete de Órbita Baja. En: Colombia. Memorias Evento: Congreso Nacional de Ingeniería Mecánica UIS 2010. Innovación e Investigación en Ingeniería. (ISBN 978 - 958 - 57001 - 0 - 9), Bucaramanga Santander 9-12 Noviembre 2010. Universidad Industrial de Santander. Bucaramanga. Colombia. 2010.

16 Quiñonez, Diego; Lizcano, Yamit; Vasquez, Cristian; Maldonado, Jorge; Portocarrero, Julián. Construcción y Evaluación de una Tobera a Escala Menor en Material Compuesto para de Cohete de Órbita Baja. En: Colombia. Memorias Evento: Simposio Internacional Sobre Sistemas Electromecánicos - SISEM 2011, Universidad Antonio Nariño. Bogotá. Colombia. 2011. Volumen 3 de la Revista Inge@uan. ISBN: 21450035. 2012.

17 Maldonado Villa, Jorge; Portocarrero Hermann, Julian; Acuña Lizarazo, Marlon Efren; Valbuena Cocunubo, Juan Jose. Evaluación del Comportamiento Mecánico de los Materiales Compuestos en Toberas para Cohetes de Órbita Baja. VII Congreso Internacional de Materiales (CIM 2013). 29 de Octubre al 1 de Noviembre, 2013. Medellín-Colombia.

18 Maldonado Villa, Jorge; Portocarrero Hermann, Julian; Cortes, Diego; Lopez, Antonio. Construcción y Desarrollo de Una Tobera a Escala Real para Cohetes de Orbita Baja. II Simposio Internacional Sobre Sistemas Electromecánicos (SISEM 2013). 1 al 2 de Noviembre, 2013. Barranquilla-Colombia

19 Maldonado Villa, Jorge; Portocarrero Hermann, Julian; Cortes, Diego; Acuña, Marlon Efren; Valbuena, Juan José. Proceso de Desarrollo de un Material Ablativo y Estructural basado en Materiales Compuestos para Toberas de Cohete en la EMAVI. V Congreso Internacional en Ciencia y Tecnología CICTA 2014. 18 al 20 de Noviembre 2014. Bogotá - Colombia. 
Maldonado Villa, Jorge. "Montaje de refuerzos de garganta para tobera de un cohete de órbita baja" En: Colombia. 2014. ICARO. ISSN: 0123-5818 p.69 - 77 v.18

21 Portocarrero H., Julián; Maldonado Villa, Jorge. "Evaluación Del Comportamiento Mecánico de Los Materiales Compuestos en Toberas para Cohetes de Orbita Baja". Colombia. 2014. Revista Colombiana De Materiales. ISSN: 2256-1013 p.303 - 309 v.5. Portocarrero Hermann, Julián; Maldonado Villa, Jorge. "9 Evaluación del comportamiento térmico de materiales compuestos de matriz polimérica en prototipos de toberas para cohetes de órbita baja". Colombia Revista Científica "General José María Córdova" ISSN: 1900-6586 ed.: Editorial Planeta v.12 fasc.13 p.275 - 290,2014 Nakka Richard, Experimental Rocketry, Mecanizado de Toberas para cohetes [en línea]. 1a ed. Canadá, Julio 2006: [Julio de 2006, última consulta: 11 marzo de 2015]. Internet: <http://www.escull.net/spanishdocs/rnakka/Mecanizado\%20de\% 20toberas\%20para\%20cohetes.pdf

24 Acuña Lizarazo, Marlon Efrén; Valvuena Cocumbo, Juan José. Evaluación del comportamiento mecánico de los materiales compuestos en toberas de cohete de órbita baja. Santiago de Cali, Colombia, 2012. Proyecto de Grado Ingeniero Mecánico, Director Ph.D. Jorge Maldonado Villa, Escuela Militar De Aviación. Facultad de Ingeniería Mecánica. [Octubre 4 de 2012].

25 Lizcano Gomez, Yamit Alfredo; Vazquez Barrero, Cristián. Construcción de prototipos de tobera de un cohete de órbita baja y su análisis térmico estructural. Santiago de Cali, Colombia, 2010. Proyecto de Grado Ingeniero Mecánico, Director Ph.D. Jorge Maldonado Villa, Escuela Militar De Aviación. Facultad de Ingeniería Mecánica. [Junio 4 de 2010].

26 Huzel, Dieter K.; Huang, David H. Modern engineering for design of liquid-propellant rocket engines. Published by the American Institute of Aeronautics and Astronautics AIAA. Washington DC. USA. Volume 147. Table 2-9. P. 50. (1992).

27 Hisaichi Ohnabe, Shoju Masaki, Masakazu Onozuka, Kaoru Miyahara, Tadashi Sasa. Potential application of ceramic matrix composites to aero-engine components. Japan, Composites: Part A 30 (1999) 489-496. Febrero 1999. [Febrero 19 de 1999, última consulta: 13 marzo de 2015]. 1999 Elsevier Science Ltd.

28 SAYLES, David C. Method of Manufacturing Hybrid Fiber-Reinforced Composite Nozzle Material. Patent 5525372. AL, UNITED STATES, assigned to The United States of America as represented by the Secretary of the Army. (2003).

29 Ritland Marcus A; Readey Dennis W; Sibold Jack; Rulis Dean Golden. Method for Making Ceramic-Metal Gradient Composites. Patent 5525374. CO, UNITED STATES assigned to Golden Technologies Company. (2003). 